Cahiers de recherches médiévales

\title{
Le cycle iconographique perdu de l'Epistre Othea de Christine de Pizan
}

Le cas des manuscrits Beauvais, BM 09 et Oxford, Bodleian Library, Bodley 421

\section{Anne-Marie Barbier}

\section{OpenEdition}

\section{Édition électronique}

URL : https://journals.openedition.org/crm/11012

DOI : $10.4000 / \mathrm{crm} .11012$

ISSN : $1955-2424$

\section{Éditeur}

Honoré Champion

\section{Édition imprimée}

Date de publication : 10 décembre 2008

Pagination : 279-299

ISSN : 1272-9752

Référence électronique

Anne-Marie Barbier, "Le cycle iconographique perdu de l'Epistre Othea de Christine de Pizan », Cahiers de recherches médiévales [En ligne], 16 | 2008, mis en ligne le 15 décembre 2011, consulté le 15

décembre 2022. URL : http://journals.openedition.org/crm/11012; DOI : https://doi.org/10.4000/crm. 11012 


\section{ris}

\section{Le cycle iconographique perdu de l'Epistre Othea de Christine de Pizan : le cas des manuscrits Beauvais, BM 09 et Oxford, Bodleian Library, Bodley 421}

Abstract: The text of the Epistre Othea, composed ca 1400, was copied out in the manuscripts $B N F, F r .606$ and BL, Harley 4431, illuminated under the supervision of Christine de Pizan who intended them for wealthy patrons. The iconographical cycles that decorate these lavish manuscripts offered some patterns, which were reused in several exemplars of the Epistre. Interestingly enough, the manuscripts kept in Beauvais and Oxford enclose numerous drawings that present noticeable iconographic similarities, which differ from the miniatures that illustrate the presentation copies.

In this paper, I wish to examine the reasons why I think there existed an iconographical cycle, partly distinct from the cycles preserved in the manuscripts Français 606 and Harley 4431. I will also attempt to indicate the usefulness of the study of this very cycle which, I will argue, enlightens the meaning of the miniatures that Christine chose ${ }^{1}$.

Résumé : Le texte de l'Epistre Othea, composé vers 1400, a été copié dans les manuscrits $B N F, F r .606$ et BL, Harley 4431, enluminés sous la direction de Christine de Pizan qui les destinait à de riches mécènes. Les cycles iconographiques décorant ces somptueux manuscrits ont offert des modèles, qui ont été repris dans plusieurs exemplaires de l'Epistre. Néanmoins, les manuscrits conservés à Beauvais et Oxford renferment de nombreux dessins présentant des similitudes iconographiques marquées, qui diffèrent des miniatures illustrant les manuscrits de présentation.

Dans cet article, nous nous proposons d'examiner les raisons pour lesquelles nous pensons qu'il a dî exister un cycle iconographique, partiellement distinct des cycles conservés dans les manuscrits Français 606 et Harley 4431. Nous tenterons également de souligner l'intérêt de l'étude de ce cycle qui, nous semble-t-il, éclaire la signification des miniatures que Christine a choisies.

Composée vers 1400, l'Epistre Othea est la première œuvre didactique dans laquelle Christine de Pizan s'adresse aux jeunes chevaliers et aux princes, dans un souci d'éducation morale et de paix civile. La cinquantaine de manuscrits et les incunables qui nous sont parvenus attestent le succès remporté par cette œuvre au cours du XV $\mathrm{XV}^{\mathrm{e}}$ siècle.

Les manuscrits Français 606 et Harley 4431, conservés à la Bibliothèque nationale de France et à la British Library², ont été produits sous la direction de

\footnotetext{
1 Nous remercions Vanessa Alayrac, Maître de Conférences au Département des Études anglaises de l'Université Charles-de-Gaulle Lille 3, de la relecture de cet abstract.

${ }^{2}$ Paris, Bibliothèque nationale de France et Londres, British Library, Harley 4431. Pour les notices, voir: G. Mombello, La tradizione manoscritta dell'Epistre Othea: Prolegomeni all'edizione del testo, Turin, Accademia delle Scienze, 1967, p. 13-23, 199-210.
}

Cahiers de Recherches Médiévales, 16, 2008 
l'auteur qui dédia le premier au duc d'Orléans ${ }^{3}$ et le second, à la reine Isabeau de Bavière ${ }^{4}$. Leurs cycles complets d'illustrations ont donné naissance à une tradition iconographique dont l'influence est perceptible sur les miniatures de plusieurs exemplaires. Cependant, les manuscrits conservés à la Bibliothèque municipale de Beauvais et à la Bodleian Library d'Oxford ${ }^{5}$ renferment de nombreuses illustrations comportant de profondes similitudes iconographiques, alors qu'elles se distinguent de celles qui sont contenues dans les manuscrits de présentation. Ce double constat fait naître une interrogation sur le modèle copiés.

À partir de l'analyse de quelques illustrations appartenant aux manuscrits Beauvais, BM 09, Oxford, Bodleian Library, Bodley 421 et BNF, Fr. 606, nous nous proposons de présenter les fondements que nous avons apportés à l'hypothèse de l'existence d'un cycle iconographique, partiellement distinct des cycles conservés, ayant illustré un manuscrit aujourd'hui perdu'. Nous chercherons à définir l'apport de l'étude de ce cycle à l'interprétation des miniatures du manuscrit Français 606. Notre étude débute par un état de la recherche ${ }^{8}$.

\section{Les cycles d'illustrations de l'Epistre Othea}

Cinq exemplaires enluminés de l'Epistre Othea remontant aux deux premières décennies du $\mathrm{XV}^{\mathrm{e}}$ siècle nous ont été transmis. À la différence du manuscrit BNF, Fr. 848, sans doute le plus ancien, qui ne contient que l'Epistre, trois des quatre autres exemplaires appartiennent à des recueils conçus par Christine

\footnotetext{
${ }^{3}$ Le manuscrit Français 606, préparé à l'intention de Louis d'Orléans, a été acquis par Jean de Berry, vers 1408, après l'assassinat du duc d'Orléans. Voir: M. Meiss et S.O. [Smith], «The bookkeeping of Robinet d'Estampes and the Chronology of Jean de Berry's Manuscripts ", Art Bulletin, 53, $\mathrm{n}^{\circ}$ 2, 1971, p. 228, 233 ; J. Laidlaw, "Christine de Pizan as Publisher », Modern Language Review, 82, 1987, p. 52-53.

${ }^{4}$ Le manuscrit Harley 4431 a été probablement offert à la reine, en janvier 1414. Voir: J. Laidlaw, «The Date of the Queen's Ms (London, British Library, Harley Ms 4431) ». Cet article, fondé sur une communication donnée au colloque de Salzbourg en juillet 2003, est publié actuellement sur le site Internet: www.pizan.lib.ed.ac.uk.harley4431date.pdf.

${ }^{5}$ Beauvais, BM, Ms 09 ; Oxford, Bodleian Library, Ms Bodley 421.

${ }^{6}$ Cet article se fonde sur notre mémoire de Master Recherche $2^{\mathrm{e}}$ année d'Histoire de l'Art, ayant porté sur l'Étude comparée des manuscrits de l'Epistre Othea, Lille, BM 175 et Oxford, Bodley 421, préparé sous la direction de Monsieur Christian Heck, Membre senior de l'Institut Universitaire de France, Professeur à l'Université Charles-de-Gaulle Lille 3 (centre IRHIS, UMR 8529), et qui a été soutenu le 29 juin 2006. Nous avons présenté une première version de cet article lors du $42^{\mathrm{e}}$ Congrès international d'Études médiévales qui s'est tenu à Kalamazoo (Michigan) en mai 2007 et bénéficié des remarques qui ont animé le débat qui a suivi. Nous remercions vivement Monsieur le Professeur Christian Heck des commentaires formulés sur la première version de cet article. Nous préparons sous sa direction une thèse sur L'illustration de l'Epistre Othea de Christine de Pizan en France et dans les anciens PaysBas, au XV siècle.

${ }^{7}$ Par l'expression «cycle iconographique perdu», nous désignons le cycle d'illustrations qui se reflète dans les manuscrits de Beauvais et Oxford, et qui était probablement contenu dans un manuscrit aujourd'hui perdu.

${ }^{8}$ Nous limitons l'état de la question aux recherches relatives aux cycles d'illustrations de l'Epistre Othea, conçus sous la direction de Christine de Pizan.
} 
pour présenter ses écrits ${ }^{9}$, ce qui était également le cas, à l'origine, du manuscrit Français $606^{10}$.

Les cycles complets de miniatures qui ornent les manuscrits Français 606 et Harley 4431 ont retenu l'attention des chercheurs. Dès 1937, dans un article relatif à l'illustration des manuscrits de Christine de Pizan, Lucie Schaëfer a présenté une description précise des cent une miniatures illustrant le texte de l'Epistre transcrit dans le manuscrit Harley $4431^{11}$. D'autre part, dans l'étude consacrée à la peinture française à l'époque du duc de Berry ${ }^{12}$, Millard Meiss a précisé les liens de parenté unissant les cycles d'illustrations des manuscrits Français 606 et Harley 4431, à partir de la datation de textes contenus dans le manuscrit londonien. Le cycle iconographique inclus dans le premier manuscrit cité, exécuté vers 1405-1408, a servi de modèle à celui du manuscrit Harley $4431^{13}$.

Plusieurs chercheurs ont mis en lumière l'implication de l'auteur dans la préparation de ces deux manuscrits. Christine a contrôlé la transcription du texte dans le manuscrit Français $606^{14}$ et elle a, elle-même, copié le texte de l'Epistre dans le manuscrit Harley $4431^{15}$. L'auteur a également participé à la conception des enluminures des deux manuscrits ${ }^{16}$. Leur cycles d'illustrations, présentant de profondes similitudes, exécutés par des maîtres différents, reflètent un " programme », sans doute défini par Christine.

Dans le manuscrit Harley 4431 rassemblant trente œuvres de Christine, cent une des cent trente-deux miniatures illustrent le texte de l'Epistre. La richesse exceptionnelle des cycles iconographiques de ces deux exemplaires de présentation est le fruit d'une lente maturation, accomplie entre 1400 et 1408 environ. Les

${ }^{9}$ Chantilly, Musée Condé, Mss 492-493 ; Paris, BNF, Fr. 12779; Londres, British Library, Ms Harley 4431.

${ }^{10}$ Une majorité de chercheurs s'accordent à penser que le manuscrit BNF, Fr. 606 faisait partie initialement d'un recueil qui comprenait les textes contenus dans les manuscrits de la Bibliothèque nationale portant les cotes 835, 606, 836 et 605. Voir: M. Roy, Euvres poétiques de Christine de Pizan, 3 vol., Paris, Firmin Didot (Société des anciens textes français), 1886, I, p. v-xii ; Mombello, op. cit., p. 16-18 ; J. Laidlaw, art. cit. (1987), p. 52-59.

${ }^{11}$ L. Schaëfer, «Die Illustrationen zu den Handschriften der Christine de Pizan », Marburger Jahrbuch füe Kunstwissenschaft, 10, 1937, p. 130-141.

${ }_{12}$ M. Meiss, French Painting in the Time of Jean de Berry. The Limbourgs and their contemporaries, 2 vol., Londres, Thames and Hudson, [New-York], Pierpont Morgan Library, 1974, I, p. 23-41.

${ }^{13}$ Meiss, op. cit., I, p. 37,440, n. 148

${ }^{14}$ C'est la main R qui a transcrit le texte du manuscrit Français 606. Voir : G. Ouy et C. Reno, "Identification des autographes de Christine de Pizan », Scriptorium, 34, 1980, p.225.

${ }_{15}$ Sur la question des manuscrits autographes de Christine, voir: C. C. Willard, «An Autograph Manuscript of Christine de Pizan?», Studi Francesi, 27, 1965, p. 452-457; G. Ouy et Reno, art. cit., p. 221-238 ; S. Hindman, " The composition of the Manuscript of Christine de Pizan's collected Works in the British Library: A Reassessment ", British Library Journal, 9, 1983, p. 92-123; J. Laidlaw, «Christine de Pizan - An author's Progress », Modern Language Review, 78, 1983, p. 532-550.

${ }^{16}$ Meiss, op. cit., I, p. 24 ; M. A. Ignatius, « An Experiment in Literary Form », Medievalia et humanistica: studies in medieval and renaissance culture, N. S. 9, 1979, p. 127-142; S. Hindman, Christine de Pizan's Epistre Othea : Painting and Politics at the Court of Charles VI, Toronto, Pontifical Institute of Mediaeval Studies (Studies and Texts, 77), 1986, p. 140142. 
manuscrits produits par Christine jusqu'en 1403 étaient dotés d'un cycle iconographique court. Ainsi, le texte de l'Epistre contenu dans le recueil des Euvres poétiques conservé à Chantilly et daté de 1402 s'accompagne de six miniatures, tout comme celui du manuscrit Français 848 renfermant la version A du texte ${ }^{17}$, et peutêtre offert par Christine à Louis d'Orléans en janvier $1401^{18}$. Le texte incomplet de l'Epistre, contenu dans le manuscrit BNF, Fr. 12779 que C. Reno date de 14021403, était sans doute illustré initialement de six miniatures ${ }^{19}$. Les manuscrits Français 606 et Harley 4431 sont les seuls exemplaires de présentation renfermant un cycle complet d'enluminures qui nous soient parvenus. Tous deux contiennent la version du texte dite $«$ de type $\mathrm{B} »^{20}$.

Néanmoins, cette vue d'ensemble des manuscrits enluminés conservés n'offre qu'une idée partielle de la production à laquelle Christine a œuvré. Dans son étude de la tradition manuscrite de l'Epistre, Gianni Mombello a souligné que plusieurs manuscrits de présentation comportant les dédicaces à Charles $\mathrm{VI}^{21}$, Philippe le Hardi et Jean de Berry ne nous ont pas été transmis ${ }^{22}$. S'appuyant sur des documents d'archives, M. Meiss a indiqué que deux de ces exemplaires, produits antérieurement au manuscrit Français 606, avaient été dotés d'un cycle complet d'illustrations. L'un d'eux, inscrit par Robinet d'Estampes sur la liste des livres acquis par Jean de Berry depuis l'inventaire dressé en 1402-1403, lui avait été offert par Christine vers 1404-1406 ${ }^{23}$. Décrit comme «très bien historié », il avait cependant été prisé au prix minime de cinquante sols ternois, après la mort du duc. À partir de ce constat, l'historien de l'art a déduit que ce manuscrit était probablement illustré de dessins ${ }^{24}$.

D'autre part, des chercheurs ont avancé l'hypothèse d'un cycle iconographique que Christine de Pizan aurait conçu dans les premières années du $\mathrm{XV}^{\mathrm{e}}$ siècle. A propos du manuscrit conservé à Cambridge ${ }^{25}$, Sandra Hindman évoque la probable existence, parallèlement à celle des manuscrits destinés au milieu princier, de manuscrits illustrés, destinés à un large public ${ }^{26}$. Par ailleurs, dans sa thèse consacrée à la réception de l'Antiquité dans les principaux manuscrits

\footnotetext{
${ }^{17}$ Mombello, op. cit., p. 29.

${ }^{18}$ Mombello, op. cit., p. 317.

${ }^{19}$ Il reste actuellement quatre miniatures. Sur la datation du manuscrit, voir : C. Reno, «Les Enseignements moraux: Good advice for Several generations », 2005, p. 1. Cet article est actuellement en ligne sur le site : www.pizan.lib.ed.ac.uk/morauxnov05. pdf. Nous voudrions remercier Madame Christine Reno, Professeur à Vassar College, New York, pour les suggestions formulées à propos du texte de la communication présentée à Kalamazoo.

${ }^{20}$ Mombello, op. cit., p. 21-22.

${ }^{21}$ Selon J. Laidlaw, il s'agit de la dédicace à Henri IV, roi d'Angleterre. Voir : Laidlaw, art. cit. (1983), p. 544.

${ }^{22}$ Mombello, op. cit., p. 317. Sur la datation des dédicaces, voir : Laidlaw, art. cit. (1983), p. 543-546.

${ }_{23}$ J. Guiffrey, Inventaires de Jean, duc de Berry : 1401-1413, Paris, E. Leroux, 1894-1896, I, p. 249, n' 949 ; M. Meiss et S. O. [Smith], art. cit., p. 227, 233 ; Laidlaw, art. cit. (1983), p. $545-546$.

${ }^{24}$ Meiss, op. cit.(1974), I, p. 439-440, n. 135.

${ }^{25}$ Epistre Othea, Cambridge, Newnham College, Ms 05.

${ }^{26}$ Hindman, op. cit. (1986), p. 140-142.
} 
enluminés de l'Epistre Othea ${ }^{27}$, Charlotte Schoell-Glass explique les similitudes iconographiques observées sur quelques miniatures des manuscrits de Beauvais et Oxford, par l'existence d'un cycle iconographique dont elle postule la présence dans un exemplaire de présentation aujourd'hui perdu, qui aurait été offert par Christine à Philippe le Hardi, avant $1404^{28}$.

Nous nous somme proposé de fonder la première partie de cette hypothèse, au moyen d'une comparaison systématique entre les illustrations des manuscrits Beauvais, BM 09, Oxford, Bodley 421 et celles du manuscrit Français $606^{29}$. Nous avons analysé méthodiquement les miniatures comportant des similitudes iconographiques dans les deux premiers manuscrits cités, leurs relations avec celles du manuscrit Français 606 et proposé une explication des similitudes constatées. Dans cet article, la présentation des résultats obtenus s'appuie sur l'étude de trois exemples.

\section{L'existence d'un cycle iconographique perdu de l'Epistre Othea}

Le texte de l'Epistre, copié sur les feuillets de parchemin du manuscrit Beauvais, BM 09, probablement vers $1410-1420^{30}$, a perdu sa dédicace. Il est illustré d'un cycle fragmentaire de quarante-deux dessins tracés à la plume et à l'encre noire, sans doute exécutés lors de la décennie suivante. La présence d'espaces réservés ${ }^{31}$ indique qu'un cycle iconographique complet était prévu initialement. L'observation des miniatures permet de déceler l'usage de procédés de reproduction mécanique, vraisemblablement des poncifs ${ }^{32}$.

Dans le manuscrit Oxford, Bodley 421, le texte de l'Epistre contient la dédicace à Philippe le Hardi ${ }^{33}$. Il est illustré d'un cycle complet de cent un dessins

${ }^{27}$ C. Schoell-Glass, Aspekte der antiken rezeption in frankreich und Flandern im 15. Jahrhundert : Die Illustrationen der Epistre Othea von Christine de Pizan, Hambourg (thèse, Université de Hambourg, (1986), [s. 1.], [s. n.], 1993.

${ }^{28}$ Schoell-Glass, op. cit., p. 112.

${ }^{29}$ En raison des similitudes iconographiques que présentent les miniatures étudiées dans cet article dans les manuscrits Français 606 et Harley 4431, nous prenons seulement en compte le premier manuscrit, en indiquant en note les variations iconographiques mineures relevées dans le second.

${ }^{30}$ Pour la notice du manuscrit, voir: Mombello, op. cit., p. 124-129. Le manuscrit renferme également le Livre de Mélibée et de Prudence, trad. par Renaut de Louhans du Liber consolationis et consilii d'Alberto da Brescia.

${ }^{31}$ Selon la définition de D. Muzerelle, un espace « réservé » est un espace laissé en blanc, en vue de l'exécution différée d'une miniature, d'une initiale ou d'une rubrique. Voir: D. Muzerelle, Vocabulaire codicologique : répertoire méthodique des termes français relatifs aux manuscrits, Paris, éd. CEMI (Rubricae), 1985, p. 333.

${ }^{32}$ Sur les techniques de reproduction mécanique utilisées dans les deux manuscrits, voir le texte de la communication présentée au $132^{\mathrm{e}}$ Congrès du Comité des travaux historiques et scientifiques: A.-M. Barbier, «La parenté iconographique de deux manuscrits de l'Epistre Othea de Christine de Pizan (Beauvais, Bib. Mun. 09 et Oxford, Bodleian Library, Bodley 421). Étude des procédés de reproduction mécanique». À paraître dans les Actes $d u 132^{e}$ Congrès national des sociétés historiques et scientifiques (Arles, avril 2007), CTHS.

${ }^{33}$ Pour la notice du manuscrit, voir: Mombello, op. cit., p. 225-232. Le manuscrit d'Oxford renferme également trois textes attribués à Alain Chartier. 
aquarellés, réalisés par plusieurs miniaturistes du Nord de la France ${ }^{34}$. Leur exécution peut être datée de 1467-1477, à partir des costumes reflétant la mode en usage à la cour de Charles le Téméraire. Le support papier présente de nombreuses traces matérielles laissées par l'usage de plusieurs procédés de reproduction mécanique : stylet, pointe métallique, poncifs ou cartons perforé ${ }^{35}$

\section{Oxford \\ Les similitudes iconographiques dans les manuscrits de Beauvais et}

L'observation comparée des deux manuscrits met en présence de nombreuses similitudes iconographiques: les figurations de Narcisse et d'Athamas en témoignent.

Dans les deux manuscrits, Narcisse est figuré seul (Ill. 1 et 2), accoté au rebord d'une «fontaine »- source aménagée par l'homme - qui se découpe sur le versant d'un coteau. Portant une corne de chasse en bandoulière, il est absorbé dans la contemplation de son reflet à la surface de l'eau. La similitude des motifs iconographiques est renforcée par l'identité du schéma de composition.

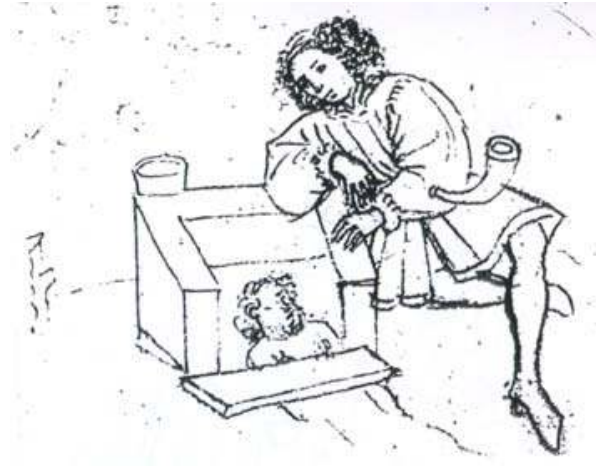

1-Epistre Othea, Beauvais, BM 09, fol. $10^{\mathrm{r}}$, Narcisse à la fontaine.

(C) Beauvais, Bibliothèque municipale.

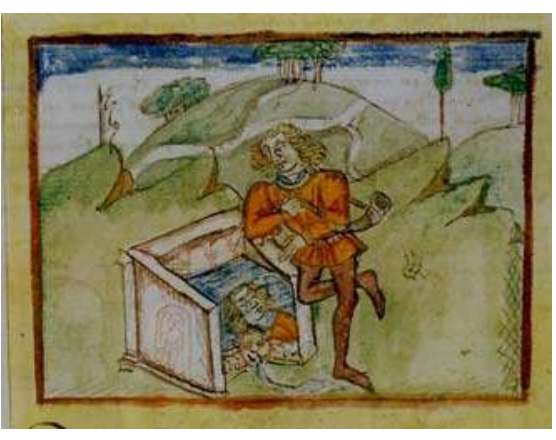

2- Epistre Othea, Bodleian Library, Ms Bodley 421, fol. $18^{\mathrm{r}}$, Narcisse à la fontaine. (C) Bodleian Library.

Ces deux types de similitudes s'observent également dans les illustrations figurant Athamas (Ill. 3 et 4). La scène, encadrée par une architecture à arcades, présente le roi debout, portant une couronne. Les bras écartés, il serre le cou de deux enfants nus. À ses pieds est étendue sa femme Ino, coiffée d'une couronne. Campée sur le seuil de l'édifice, Tisiphone brandit un serpent dans chaque main.

\footnotetext{
${ }^{34}$ L'influence du miniaturiste lillois portant le nom de convention «Maître de Wavrin » est perceptible sur plusieurs miniatures du manuscrit.

${ }^{35}$ Voir notre note 32.
} 

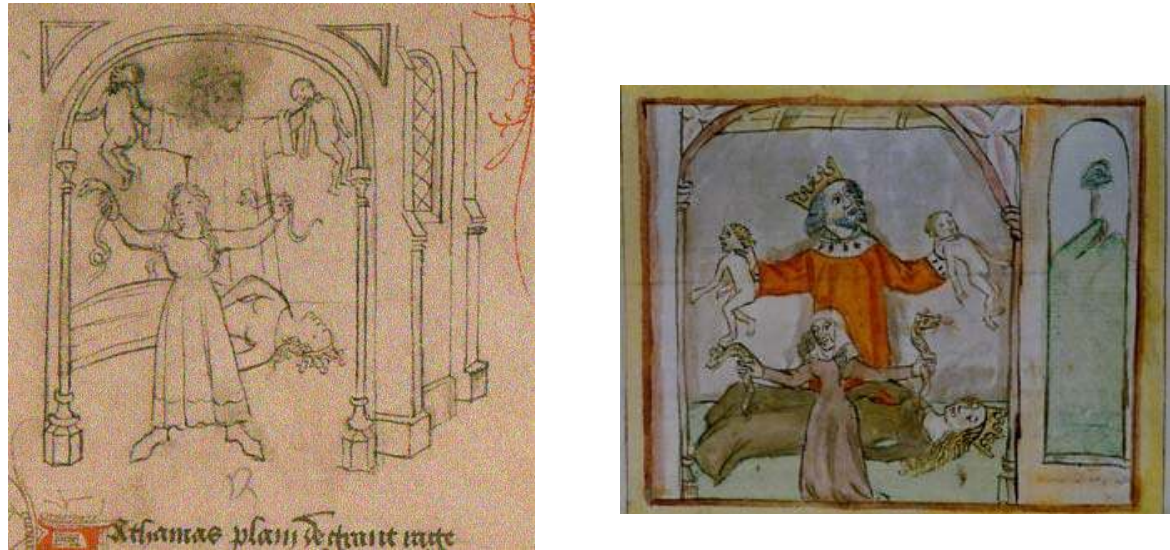

3-Epistre Othea, Beauvais, BM, 09, 4-Epistre Othea, Oxford, Bodleian fol. $10^{\mathrm{r}}$, Athamas furieux. (C) Beauvais, Library, Bodley 421, fol. $18^{\mathrm{v}}$, Athamas Bibliothèque municipale.

furieux. (C) Bodleian Library, Université d'Oxford.

L'étude comparée systématique des quarante-deux illustrations du manuscrit de Beauvais et de celles qui leur correspondent dans celui d'Oxford nous a permis de constater que trente-six d'entre elles comportent des similitudes iconographiques plus ou moins profondes. Cependant, celles-ci n'entraînent pas une identité absolue. La presque totalité des illustrations laisse voir des différences iconographiques mineures, relatives aux mêmes types d'éléments. Ainsi, dans le second manuscrit, les costumes ont été mis au goût du jour. Le pourpoint court aux épaules rembourrées, que Narcisse porte sur des chausses moulantes, reflète la mode en usage à la cour de Bourgogne, dans les années 1467-1477. Par ailleurs, les paysages, montagneux ou vallonnés, ont été librement esquissés par les miniaturistes. En outre, les architectures à deux dimensions du manuscrit d'Oxford contrastent avec les représentations architecturales qui, dans celui de Beauvais, suggèrent la profondeur de l'espace. Dans chaque manuscrit, le miniaturiste a interprété le modèle copié en fonction de son propre style et de la maîtrise qu'il possède de son art.

Comparaison des illustrations des manuscrits de Beauvais et Oxford présentant des similitudes à celles du manuscrit Français 606

L'existence d'un nombre élevé d'illustrations présentant des similitudes iconographiques dans ces deux manuscrits soulève la question de leurs rapports avec celles du manuscrit Français $606^{36}$. L'étude comparée systématique de ces trente-six illustrations dans les trois manuscrits nous a permis d'établir que vingt-quatre d'entre elles diffèrent de celles du Français 606.

${ }^{36}$ Les cycles iconographiques courts complets contenus dans les premiers manuscrits de Christine ne comportent pas d'illustrations des textes dont les enluminures ont été étudiées dans les manuscrits de Beauvais et Oxford. 
Ces différences relèvent de trois types: les variations iconographiques, les écarts liés à la présence de motifs iconographiques différents et les ruptures, découlant principalement du choix d'épisodes distincts d'une même histoire.

Les représentations de Narcisse offrent un exemple des premières. Dans les trois manuscrits, le jeune homme est figuré seul, penché au-dessus de l'eau d'une fontaine, fasciné par sa propre image. La nymphe Echo n'est pas représentée (Ill. 1, $2,5)^{37}$. Deux différences séparent les illustrations des manuscrits de Beauvais et Oxford de celle du manuscrit Français 606.

Dans les deux premiers manuscrits étudiés (Ill. 1 et 2), la scène se déroule dans un espace ouvert, illimité, alors que dans le manuscrit Français 606 (Ill. 5), elle prend place dans une forêt, dans un locus amœnus, lieu ombragé et accueillant, propice aux rencontres amoureuses. Dans ce manuscrit, le motif iconographique évoquant, de façon discrète, les circonstances dans lesquelles le jeune homme s'est arrêté près de la fontaine - la corne de chasse - est renforcé par la représentation d'un cerf, de deux chiens, dont un lévrier, qui traversent la forêt en courant alors que des oiseaux sillonnent le ciel. La scène est située explicitement dans un contexte cynégétique ${ }^{38}$.

Les figurations d'Athamas illustrent le second type de différences. Dans les trois manuscrits, la miniature qui ouvre le texte [17] représente cinq personnages : le roi Athamas, Tisiphone - l'une des Furies - et ses trois victimes (Ill. 3, 4 et 6$)^{39}$. L'illustration relie la folie du roi et les maléfices de Tisiphone.

\footnotetext{
${ }^{37}$ Les enlumineurs de l'Ovide Moralisé représentent souvent sur la même miniature deux séquences successives : Écho rejetée par Narcisse et Narcisse contemplant son image.

${ }^{38} \mathrm{Au}$ folio $104^{\mathrm{r}} \mathrm{du}$ manuscrit Harley 4431, la miniature figure un cerf poursuivi par un chien, arrêté dans sa course par un animal, partiellement masqué par les feuillages.

39 Reprenant le livre IV des Métamorphoses d'Ovide (v. 481-542), l'auteur de l'Ovide Moralisé présente la folie d'Athamas et d'Ino comme un acte de vengeance de la déesse Junon, jalouse de sa rivale, Sémélé. Le dieu Bacchus, fils de Sémélé et de Jupiter, ayant été élevé par sa tante Ino et son époux Athamas, Junon descendit aux Enfers où elle obtint le concours de Tisiphone, afin de les punir en les frappant de folie. Voir: Ovide Moralisé: Poème du commencement du quatorzième siècle, éd. C. de Boer, Amsterdam, J. Müller (Verhandeligen der Koninklijke Akademie van wetenschappen te Amsterdam. Afdeeling letterkunde nieuwe Reeks, deel 15, 21, 30, 37), 5 vol., 1915-1938, liv. IV, p. 93-05, v. 38763963.
} 


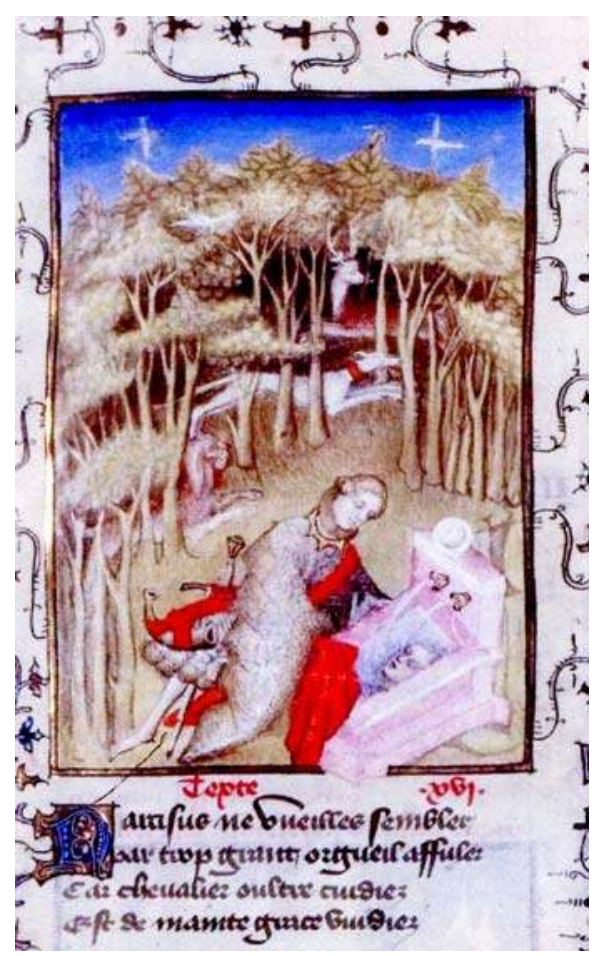

5- Epistre Othea, Paris, BNF, Fr. 606, fol. $10^{\mathrm{r}}$, Narcisse à la fontaine.

(C) Bibliothèque nationale de France.

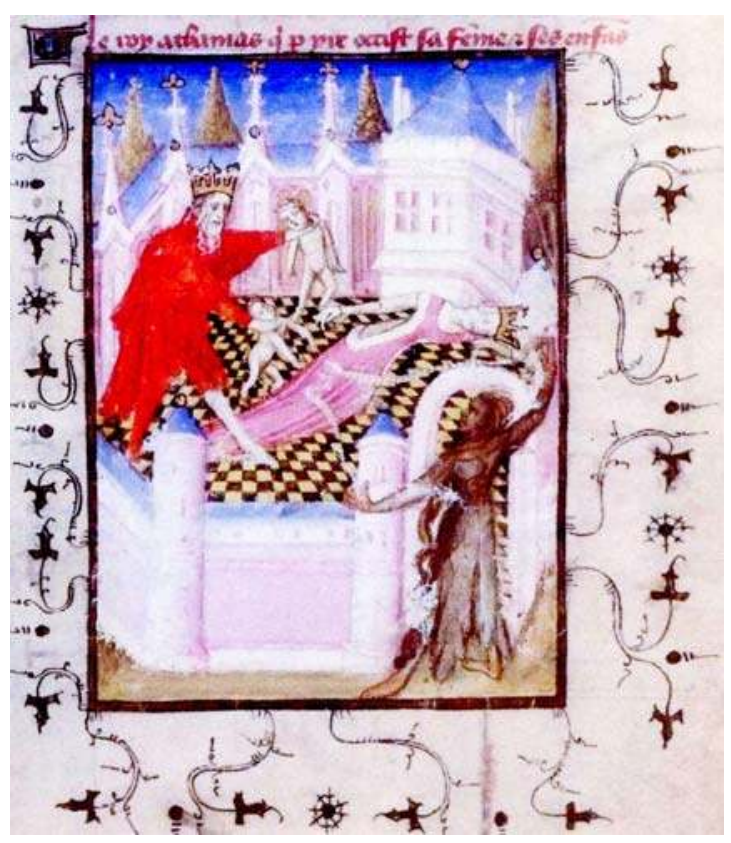

6- Epistre Othea, Paris, BNF, Fr. 606, fol. $10^{\mathrm{r}}$, Athamas mettant à mort ses enfants. (C) Bibliothèque nationale de France.

Dans les manuscrits de Beauvais et Oxford, les miniaturistes ont représenté la crise de folie furieuse du roi Athamas, à l'intérieur d'une demeure anonyme à pan de mur avant coupé (Ill. 3 et 4). Au troisième plan, figuré debout, en position frontale, le roi Athamas portant une couronne, empoigne par le cou deux petits enfants nus, alors que son épouse Ino gît sur le sol. La disposition des figures sur deux plans parallèles et l'analogie des postures de Tisiphone et Athamas suggèrent le caractère diabolique de l'action de ce dernier.

Dans le manuscrit Français 606, l'illustration présente un écart iconographique majeur découlant du parti de représenter la scène au sein d'une enceinte fortifiée aux murailles surbaissées (Ill. 6). Depuis l'extérieur de celle-ci, Tisiphone vient de lancer deux serpents dans la direction du roi. À l'intérieur des remparts, Athamas, vêtu d'une robe rouge, coiffé d'une couronne, se présente de trois quarts, étranglant de ses mains deux enfants nus ${ }^{40}$. Une seconde différence, relative à la composition, tient à la présentation de la scène en contre-plongée, qui transforme la crise de folie furieuse du roi Athamas en un spectacle tragique.

${ }^{40} \mathrm{Au}$ folio $104^{\mathrm{r}} \mathrm{du}$ manuscrit Harley 4431 , les deux enfants sont de sexe masculin. 
La figuration des pratiques maléfiques provoquant la folie d'Athamas est fortement expressive. Le motif iconographique des serpents, «formes de prédilection du Diable $»^{41}$, est intégré dans une composition qui participe à la signification de l'image. Des bras écartés de Tisiphone partent deux lignes sinueuses le long desquelles progressent les deux vecteurs du mal. Elles passent par les bras du premier enfant, le bras droit d'Athamas, traversent son corps, son bras gauche et le second enfant, avant de se fermer en boucle. Cette composition des motifs iconographiques suggère l'idée que les forces maléfiques dont le roi est victime engendrent ses réactions meurtrières, de façon mécanique et inéluctable.

Les miniatures représentant Jason dans les manuscrits de Beauvais, Oxford, et dans le manuscrit Français 606 (Ill. 7, 8 et 9), témoignent du troisième type de différences, fondé sur une rupture iconographique.
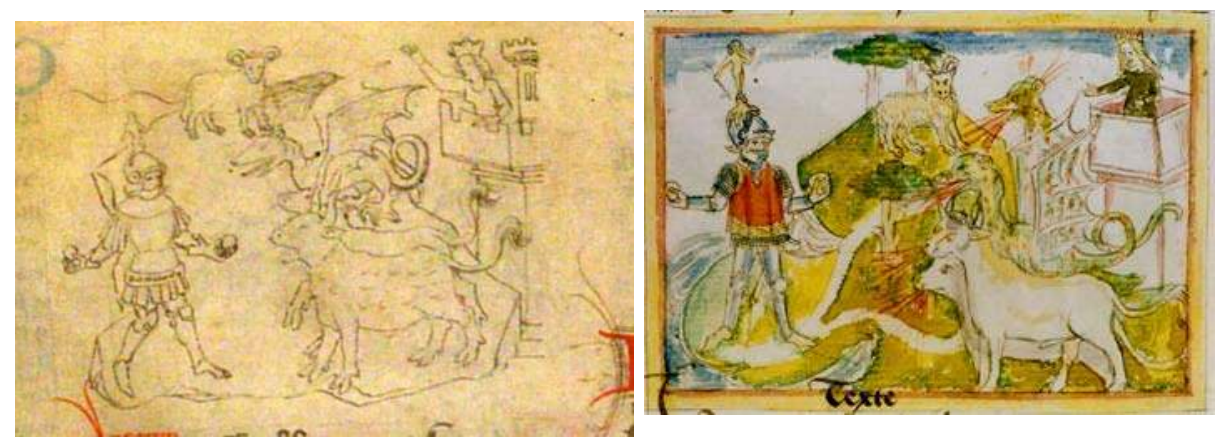

7-Epistre Othea, Beauvais, BM, fol. $29^{\mathrm{r}}$, Jason affrontant les taureaux.

09, 8-Epistre Othea, Oxford, Bodleian (C) Beauvais, Bibliothèque municipale. Library, Bodley 421, fol. $40^{\mathrm{v}}$, Jason affrontant les taureaux. (C) Bodleian Library, Université d'Oxford.

Dans les deux premiers manuscrits cités, les miniaturistes représentent le premier moment de la conquête de la Toison d'or (Ill. 7 et 8) ${ }^{42}$. À son arrivée sur l'île de Colchos, Jason fait face aux taureaux soufflant et crachant le feu. Revêtu d'une armure, coiffé d'un heaume, il est dépourvu d'épée et d'écu. Il est protégé par une statuette de divinité nue, fixée sur son heaume, qui lui sert de talisman. Il tient dans les mains des objets sphériques, des boules de glu au moyen desquelles il soumettra les taureaux à un joug ${ }^{43}$. Il les attellera ensuite à une charrue, afin de leur faire labourer le champ. Après les avoir domptés au moyen de procédés magiques, il mettra à mort le dragon qui garde le bélier et s'emparera de la toison d'or. Au

${ }^{41}$ Selon l'expression d'E. Mozzani dans Le Livre des superstitions. Mythes, croyances et légendes, Paris, Laffont (Bouquins), 1995, p. 1630.

${ }_{42}$ La conquête de la Toison d'or à laquelle fait référence le texte [54] est le but de l'expédition des Argonautes sur l'île de Colchos. Sur le récit de cette expédition dans le Roman de Troie de Benoît de Sainte-Maure, voir: M.-R. Jung, La légende de Troie au Moyen Âge, Bâle et Tübingen, Francke Verlag, 1996, p. 40-41.

${ }^{43} \mathrm{La}$ statuette de divinité protectrice et les boules de glu sont deux des procédés magiques transmis par Médée à Jason pour lui permettre d'accomplir sa mission. 
sommet d'une tour quadrangulaire, se tient la princesse Médée, coiffée d'une couronne, qui s'adresse à Jason d'un geste de la main. Par la représentation de la princesse et des procédés magiques, l'illustration évoque l'aide que Médée a apportée à Jason, rappelant la dette morale de ce dernier.

Dans le manuscrit Français 606, Christine a choisi de faire représenter le second épisode de la Conquête de la Toison d'or, le combat de Jason contre le dragon.

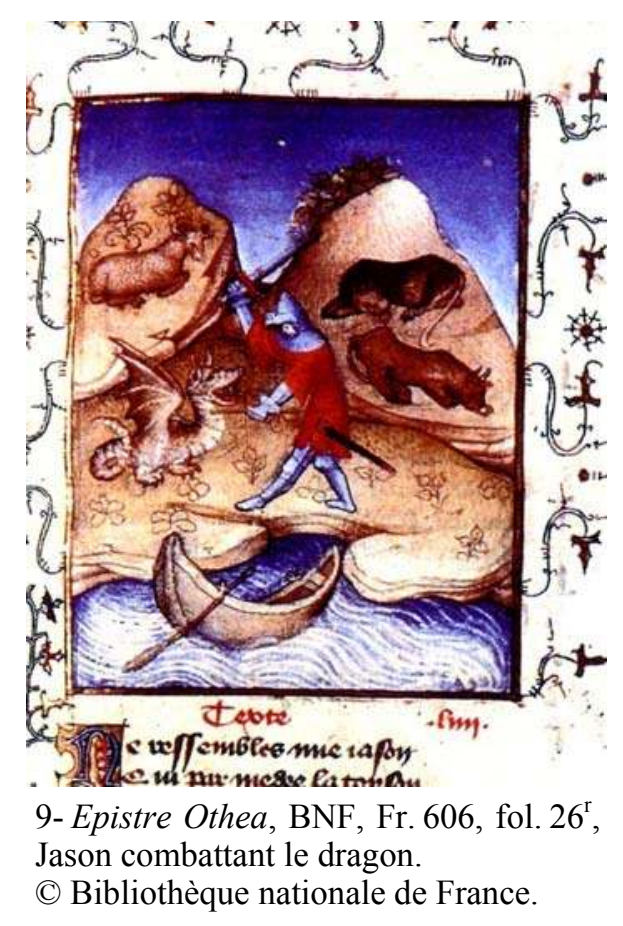

Le miniaturiste figure le «chevalier grec» sur l'île de Colchos, lors des derniers moments du combat (Ill.9). Protégé par une armure, un heaume et un écu, armé d'un épieu et d'une épée, Jason a déjà transpercé le cou du dragon de son épieu, il s'apprête à lui trancher la tête d'un coup d'épée. Médée n'est pas figurée sur la miniature. Une seconde différence porte sur l'armement dont Jason est pourvu. Sur la miniature du manuscrit Français 606, Jason se bat au moyen d'armes traditionnelles, épée et épieu. L'illustration n'évoque nullement la dette morale qu'il a contractée à l'égard de Médée.

Ainsi, les miniatures des trois manuscrits offrent deux images distinctes du héros : dans les deux premiers manuscrits étudiés, Jason doit son salut et sa victoire à l'aide apportée par Médée ; dans le troisième, le succès de sa mission repose sur sa force et son courage.

L'analyse comparée de ces triades de miniatures nous a mis en présence des trois types de différences qui séparent les vingt-quatre illustrations présentant des similitudes iconographiques dans les manuscrits de Beauvais et Oxford de celles du manuscrit Français 606. En raison de ces différences, on ne peut considérer que ces illustrations ont pour modèles celles du manuscrit Français 606.

\section{Explication des similitudes iconographiques}

Le nombre élevé de miniatures qui, dans les manuscrits de Beauvais et Oxford, comportent des similitudes iconographiques, alors qu'elles se distinguent 
des illustrations du manuscrit Français 606, incite à s'interroger sur le cycle iconographique qui leur a donné naissance.

Afin d'expliquer les similitudes observées, deux hypothèses sont envisageables. La première incline à penser que les illustrations du manuscrit de Beauvais ont été copiées dans celui d'Oxford, plus tardif; la seconde est fondée sur l'idée qu'un cycle iconographique contenu dans un manuscrit antérieur à celui de Beauvais a été utilisé comme modèle pour illustrer les deux manuscrits.

Cependant, plusieurs faits observés s'avèrent inexplicables à partir de la première hypothèse. Le manuscrit de Beauvais est doté d'un cycle fragmentaire d'illustrations, celui d'Oxford comporte un cycle complet. Comment un cycle de cent une illustrations aurait-il pu être élaboré à partir d'un modèle n'en comportant que quarante-deux? En outre, le premier manuscrit comporte des miniatures inachevées et les deux manuscrits renferment des traces laissées par les procédés de reproduction utilisés pour mettre en place le dessin sous-jacent des miniatures. Comment les miniaturistes qui ont enluminé le manuscrit d'Oxford auraient-ils pu mener à terme leurs miniatures en copiant, par des procédés mécaniques, les illustrations du manuscrit de Beauvais? Le cycle iconographique de ce manuscrit ne peut leur avoir servi de modèle. L'hypothèse la plus plausible consiste à penser que les miniatures présentant des ressemblances iconographiques dans les deux manuscrits tirent leur origine d'un modèle commun. Ce cycle iconographique, copié dans le manuscrit de Beauvais, était probablement contenu dans un manuscrit aujourd'hui disparu.

Des observations faites sur d'autres exemplaires de l'Epistre Othea renforcent cette hypothèse. Dix illustrations contenues dans le manuscrit conservé à la Bibliothèque municipale de Lille sous la cote $175^{44}$ présentent des similitudes avec des miniatures des manuscrits de Beauvais et Oxford qui se distinguent de celles du manuscrit Français 606. La miniature du folio $39^{\mathrm{v}}$ de ce manuscrit témoigne de cette parenté (Ill. 10, 11).

Quatre autres manuscrits contiennent également des illustrations inspirées par ce même cycle iconographique ${ }^{45}$. Par ailleurs, l'édition du texte de l'Epistre, publiée vers 1499 par Philippe Pigouchet sous le titre Les Cent histoires de troye, est illustrée de bois gravés, apparentés étroitement, dans leur presque totalité, aux illustrations spécifiques du cycle perdu ${ }^{46}$.

${ }^{44}$ Ce manuscrit peut être daté de 1455-1460, à partir des costumes.

45 Il s'agit des manuscrits Aylesbury, Waddesdon Manor, collection Rothschild, 08 ; Bruxelles, BR, 9092 ; Cologny-Genève, Fondation Bodmer, 49; Erlangen, BU, 2361.

${ }^{46}$ Deux exemplaires des Cent histoires de troye sont conservés à Paris : BNF, Rés. Ye 286 et Bibliothèque Mazarine, Inc. N. 1160. 

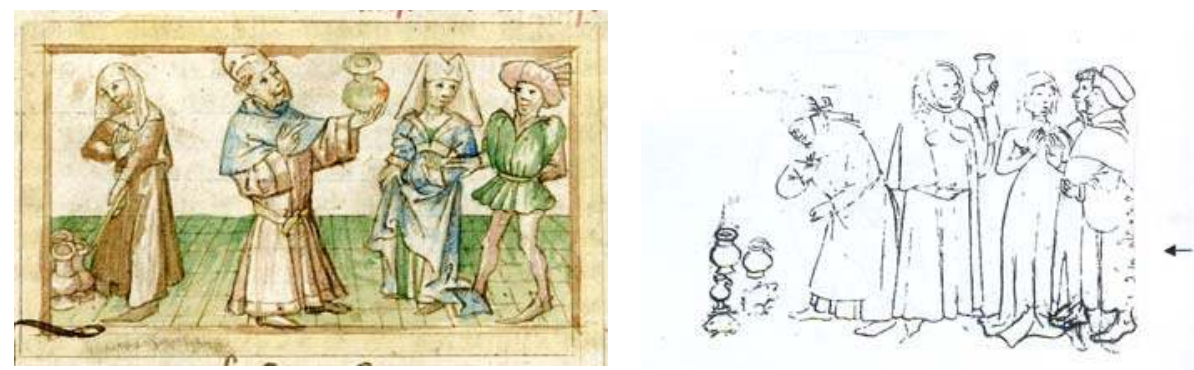

10-Epistre Othea, Lille, BM, 175, 11-Epistre Othea, Beauvais, BM, 09, fol. $39^{\mathrm{v}}$, Esculape et Circé. fol. $21^{\mathrm{v}}$, Esculape et Circé.

(C) Bibliothèque municipale de Lille.

(C) Bibliothèque municipale de Beauvais.

Les reflets de ce cycle relevés sur les illustrations de l'édition Pigouchet incitent à s'intéresser aux sources du texte imprimé, en vue de tenter d'identifier le manuscrit susceptible d'avoir contenu le cycle perdu. Les recherches menées par G. Mombello ont mis l'accent sur les liens de filiation rattachant ce texte à ceux des manuscrits de Beauvais et Cambridge ${ }^{47}$. Le philologue précise que le texte édité partage les erreurs et les variantes communes aux textes des deux manuscrits. Il en déduit que l'édition a " été tirée d'un codex peut-être perdu qui devait transmettre une leçon ${ }^{48}$ très proche de celle des deux manuscrits $\rangle^{49}$. Cependant, malgré les similitudes iconographiques entre les miniatures du manuscrit de Beauvais et les planches gravées des incunables, rien ne nous permet d'affirmer que le cycle perdu se trouvait dans le manuscrit dont le texte, transcrit dans ceux de Beauvais et Oxford, a été imprimé par Philippe Pigouchet.

La recherche dont nous avons présenté les principaux résultats nous conduit ainsi à avancer l'idée de l'existence probable d'un cycle iconographique, distinct des cycles que Christine a conçus pour les manuscrits princiers conservés, qui a exercé une influence notable sur la tradition iconographique de l'Epistre. Cette recherche laisse présentement en suspens la question de son origine. Aussi faisons-nous abstraction dans l'étude qui suit, de toute hypothèse relative à cet aspect.

\section{Intérêt de l'étude du cycle perdu}

L'étude comparative du cycle iconographique perdu qui se reflète, pensonsnous, dans les manuscrits de Beauvais et Oxford et du cycle conservé dans le manuscrit Français $606^{50}$ est susceptible d'éclairer la signification des miniatures

${ }^{47}$ Mombello, op. cit., p. 366.

${ }^{48}$ Selon D. Muzerelle, le terme «leçon» désigne chacune des formes données à un mot ou une partie de texte, dans différents manuscrits. Voir : Muzerelle, op. cit., p. 443.

${ }^{49}$ Mombello, op. cit., p. 366. 
ornant ce dernier. C'est ce que nous nous proposons de mettre en évidence en privilégiant les figures de Narcisse et Athamas ${ }^{51}$.

\section{Analyse de la figuration de Narcisse}

La comparaison des illustrations 1, 2 et 5 a souligné l'existence d'une similitude iconographique majeure dans les trois manuscrits : Narcisse est représenté seul, s'accotant au rebord d'une fontaine. Ce parti figuratif retient l'attention car il rompt avec la tradition iconographique de l'Ovide Moralisé qui se caractérise par la représentation des deux jeunes gens, sur une seule ou plusieurs miniatures.

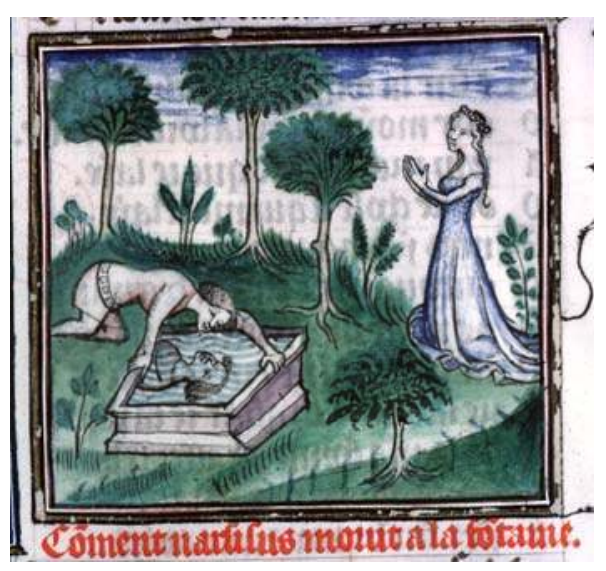

Ainsi, au folio $48^{\mathrm{r}}$ du manuscrit Lyon, BM 742, deux séquences sont figurées sur la même miniature: Écho, dédaignée par Narcisse, adressant une prière aux dieux et Narcisse, s'éprenant de sa propre image ${ }^{52}$ (Ill. 12). Ce constat suscite une interrogation sur la fidélité du texte [16] de l'Epistre à celui de l'Ovide Moralisé, source principale de Christine.

12- Ovide Moralisé, Lyon, BM 742, fol. $48^{\mathrm{r}}$, Narcisse et Écho.

(C) Bibliothèque municipale de Lyon.

Crédit photographique Bibliothèque municipale de Lyon, Didier Nicole.

Le texte de l'Epistre s'ouvre sur un portrait du jeune homme, faisant écho à celui de 1'Ovide Moralisés3. Christine caractérise Narcisse par sa beauté et son orgueil, doublé d' « oultrecuidance » :

\footnotetext{
${ }^{50}$ Cette étude pourrait être élargie au manuscrit Harley 4431, en prenant en compte les variations iconographiques mineures observées sur les illustrations correspondantes.

${ }^{51}$ Nous limitons notre étude aux deux premiers exemples, le troisième ayant fait l'objet d'une analyse dans une communication présentée lors du $132^{\mathrm{e}}$ Congrès du Comité des travaux historiques et scientifiques. Voir : A.-M. Barbier, « Représentations de la magie dans l'Epistre Othea : étude comparée du rapport texte-images dans les manuscrits Paris, BNF, Fr. 606 et Beauvais, Bibl. Mun. 09 ». À paraître dans les Actes du $132^{e}$ Congrès national des sociétés historiques et scientifiques (Arles, avril 2007), CTHS.

${ }_{52} \mathrm{Il}$ en est de même dans les manuscrits Rouen, BM, 04, fol. $81^{\mathrm{v}}$ et Arsenal, 5069, fol. $33^{\mathrm{r}}$.

${ }^{53}$ Ovide Moralisé, éd. cit., liv. III, p. 331, v. 1504-1509 : « De Narcisus le biau, le gent / [...] Mes il fu tant outrecuidiez, / Plains d'orgueil et de sens vuidiez / Qu'il perdi dou siecle la grace. »
} 
Narcisus fu un damoisel qui par sa grande beauté se esleva en si grant orgueil que il avoit en despris tous les autres. ${ }^{54}$

Cependant, la présentation de l'histoire de Narcisse, considérablement condensée $^{55}$, ne recoupe que partiellement le texte du poème. Dans celui-ci, la scène se déroulant à la fontaine est précédée d'une séquence au cours de laquelle Narcisse éconduit la nymphe Écho. À la différence de l'auteur anonyme du poème, Christine dissocie les deux scènes, en supprimant toute référence à la nymphe dans le texte $[16]^{56}$. Supposant connue l'histoire du jeune homme qui, s'étant penché au-dessus d'une fontaine pour se désaltérer, s'est abîmé dans la contemplation de sa propre image, l'auteur explique sa mort par l'orgueil :

Et pour ce que il ne prisoit se lui non, est il dit que il fu si amoureux de lui meisme que il en mourut, après que il fu mirez en la fontaine $e^{57}$.

Christine élimine ainsi le lien causal suggéré par le poème entre la plainte des femmes dédaignées par Narcisse et sa fin tragique ${ }^{58}$.

Bien que le texte [16] de l'Epistre ne précise pas les circonstances dans lesquelles Narcisse est arrivé à la fontaine, dans le manuscrit Français 606, la scène est représentée dans un décor reflétant la description que, depuis le Lai de Narcisse ${ }^{59}$, des générations d'écrivains en ont donnée (Ill.5). Guillaume de Lorris dans le Roman de la Rose ${ }^{60}$, l'auteur anonyme de l'Ovide Moralisé, Jean Froissart dans le Joli Buisson de Jonece ${ }^{61}$ présentent le jeune homme sous les traits d'un chasseur qui, accablé par la chaleur alors qu'il poursuivait un cerf, s'est arrêté en un lieu ombragé, près d'une fontaine, afin de se désaltérer. Ainsi, la mise en évidence des différences

${ }^{54}$ Christine de Pizan, Epistre Othea, éd. G. Parussa, Genève, Droz (Textes littéraires français, 517), 1999, p. 226, 1. 8-10.

${ }^{55}$ Dans l'Epistre, l'histoire de Narcisse comporte seulement vingt-huit lignes alors que quatre cent dix-sept vers lui sont consacrés dans l'Ovide Moralisé.

${ }^{56}$ Christine reprend l'histoire d'Écho dans le texte [86]. Voir : Epistre Othea, éd. cit., p. 321323.

${ }^{57}$ Epistre Othea, éd. cit., [16], p. 226, 1. 10-12.

${ }^{58}$ Dans l'Ovide Moralisé, plusieurs femmes rejetées par Narcisse - et non pas seulement Écho - demandent aux dieux de faire vivre à Narcisse un amour non réciproque. Voir : Ovide Moralisé, éd. cit., t. I, liv. III, p. 331-332, v. 1525-1543..

${ }^{59}$ Le Lai de Narcisse est un conte anonyme du XII ${ }^{\mathrm{e}}$ siècle, adapté des Métamorphoses d'Ovide. Voir: Pyrame et Thisbé, Narcisse, Philomena. Trois contes du XII siècle français imités d'Ovide, éd. et trad. E. Baumgartner, Paris, Gallimard (Folio classique, 3448), 2000. Sur ce conte, voir: E. Baumgartner, "Narcisse à la fontaine : du «conte » à l'exemple», Cahiers de Recherches Médiévales, 9, 2002, p. 133-141.

${ }^{60}$ On sait que le Roman de la Rose, écrit entre 1225 et 1278 par Guillaume de Lorris et Jean de Meun, comporte une adaptation de l'histoire d'amour non réciproque de Narcisse et Écho, tirée des Métamorphoses d'Ovide (liv. III, v. 407-510). Voir: Ovide, Métamorphoses, éd. et trad. G. Lafaye, Paris, Les Belles Lettres (Collection des universités de France), 1985, 3 vol.

${ }^{61}$ Ce poème, inclus dans les Euvres poétiques de Jean Froissart copiées dans les manuscrits BNF, Fr. 830 (fol. 155a-197d) et Fr. 831 (fol. 170b-213a), a été réédité au XX $X^{\mathrm{e}}$ siècle. Voir : Jean Froissart, Le Joli Buisson de Jonece, éd. A. Fourrier, Genève, Droz (Textes littéraires français, 222), 1975. 
textuelles entre l'Epistre Othea et l'Ovide Moralisé n'épuise pas la signification de l'illustration. On ne peut interpréter la présentation visuelle du mythe de Narcisse offerte par Christine dans le manuscrit Français 606, sans prendre en compte les liens intertextuel et visuel qui tissent la signification du texte et de l'image ${ }^{62}$.

Les allusions au contexte cynégétique contenues dans l'illustration du manuscrit Français 606 suggèrent de comparer le texte de Christine à celui du mythe ovidien de Narcisse, réécrit dans le Roman de la Rose $e^{63}$. En effet, les manuscrits du Joli Buisson de Jonece de Froissart ne comportent pas de figuration de Narcisse ${ }^{64}$; les miniatures le représentant dans les principaux manuscrits de l'Ovide Moralisé du $\mathrm{XIV}^{\mathrm{e}}$ siècle n'établissent pas de référence visuelle à son activité de chasseur, pourtant décrite dans le poème ${ }^{65}$. Seuls, certains manuscrits du Roman de la Rose évoquent ce contexte. Ainsi, au folio $11^{\mathrm{r}} \mathrm{du}$ manuscrit Oxford, Douce 371, enluminé dans un atelier parisien connu de Christine ${ }^{66}$, la miniature représentant Narcisse suggère cette activité par le motif du cheval attaché à un arbre ${ }^{67}$.

Cette évocation visuelle du contexte, commune au manuscrit Français 606 de l'Epistre Othea et à certains exemplaires du Roman de la Rose, met en évidence la relation de discordance que le texte [16] de l'Epistre entretient avec ce dernier. Guillaume de Lorris rend la nymphe Écho responsable du châtiment infligé à

\footnotetext{
${ }^{62}$ Sur l'utilité du concept d' ' intertextualité » dans l'interprétation du texte de l'Epistre Othea, voir: G. Parussa, «Le concept d'intertextualité comme hypothèse interprétative d'une œuvre », Studi francesi, 111, 1993, p. 471. L'intertextualité peut être définie par le procédé qui a donné naissance à un texte nouveau par «transposition d'énoncés pris à d'autres textes » (Julia Kristeva) ou par ses effets : "la relation de "co-présence" entre deux ou plusieurs textes » (Gérard Genette). Les liens intertextuels présents dans le texte [16] nous semblent relever d'une forme d'intertextualité non explicite fondée sur l'allusion, celle d'un énoncé «dont la pleine intelligence suppose la perception d'un lien entre lui et un autre auquel renvoie nécessairement telle ou telle de ses inflexions, autrement non recevable». Voir: G. Genette, Palimpsestes. La littérature au second degré, Paris, éd. du Seuil (Points, 257), 1982, p. 8.

${ }^{63}$ Marilynn Desmond et Pamela Sheingorn ont étudié le lien de l'illustration du folio $104^{\mathrm{r}} \mathrm{du}$ manuscrit Harley 4431 avec la seconde partie du Roman de la Rose. Nous limitons notre étude au rapport entre l'illustration choisie par Christine pour les manuscrits Français 606 et Harley 4431 et, d'autre part, le texte de Guillaume de Lorris. Voir: M. Desmond et P. Sheingorn, Myth, Montage and Visuality in late medieval Culture: Christine de Pizan's Epistre Othea, University of Michigan, Ann Arbor, 2003, p. 70-77.

${ }^{64}$ Voir les manuscrits BNF, Fr. 630 et 631.

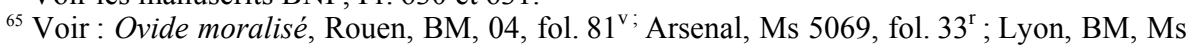
742 , fol. $48^{\mathrm{r}}$.

${ }^{66}$ Dans de nombreux manuscrits du Roman de la Rose, Narcisse est figuré seul et sans référence au contexte. C'est le cas, par exemple, dans les manuscrits BNF, Fr. 1558 (fol. 12v),

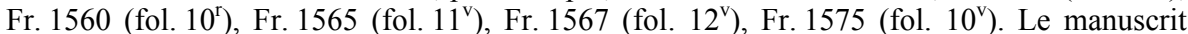
Oxford, Bodleian Library, Douce 371 a été enluminé dans le même atelier que l'exemplaire $\mathrm{du}$ Chemin de longue étude, offert par Christine de Pizan à Philippe le Hardi. Voir : Desmond et Sheingorn, op. cit., p. 72, fig. 2.16 ; p. 208.

${ }^{67}$ Ce motif iconographique a sa source dans le Lai de Narcisse : «Il met pied à terre près de la margelle de marbre et attache son cheval à un arbre. » Voir : éd. cit., p. 129.
} 
Narcisse $^{68}$, en présentant l'amour insensé du jeune homme pour sa propre image comme l'accomplissement de la prière adressée par celle-ci à Dieu afin d'obtenir vengeance $^{69}$. Cette inflexion du récit ovidien sert de support à la moralisation que Guillaume adresse, de façon déconcertante, aux dames. Leur imputant l'attitude dont Narcisse donne l'exemple, il les invite à rejeter le mode de comportement adopté par le jeune homme et à mieux aimer «leurs amis». Ainsi, éviteront-elles de susciter, par leur indifférence, la mort des hommes qui les aiment et d'encourir le châtiment divin $^{70}$. À la différence de l'auteur du Roman de la Rose, Christine tient Narcisse pour le seul responsable de sa mort.

Dans son enseignement didactique, Christine présente Narcisse comme un contre-exemple que le jeune chevalier se doit de rejeter. L'auteur prend parti en faveur de la condamnation de l'orgueil formulée dans l'Ovide Moralisé. C'est l'orgueil qui a entraîné Narcisse dans les «folies de l'amour » ${ }^{71}$ et la mort ${ }^{72}$. Nuisible à la bonne renommée ${ }^{73}$, ce vice asservit les hommes aux biens périssables, la beauté, la richesse, la puissance, « faulz miroirs de ce monde ${ }^{74} »$. Christine met en garde le chevalier chrétien contre le danger d'être « deceu par la beauté de [sa] jeunece [qui] n'est mie chose durable ${ }^{75} \gg$.

Ainsi, en accompagnant un texte qui blâme l'orgueil, d'une illustration figurant Narcisse dans un décor qui évoque celui de certaines miniatures du Roman de la Rose, l'auteur substitue sa propre moralisation à celle que Guillaume inférait de la scène de Narcisse à la fontaine. Christine a, sans nul doute, choisi cette illustration de façon intentionnelle car, en faisant figurer le type de décor de certains manuscrits du Roman de la Rose, elle fait ressortir le rejet de l'interprétation misogyne avancée par Guillaume de Lorris $^{76}$.

Les ressemblances entre les illustrations figurant Narcisse dans les trois manuscrits de l'Epistre reflètent la suppression opérée par Christine de la séquence consacrée à Écho dans l'Ovide Moralisé. En représentant Narcisse seul, ces

\footnotetext{
${ }^{68}$ Nous ne partageons pas le jugement formulé par M. Desmond et P. Sheingorn selon lesquelles «Echo joue un rôle mineur dans la version de l'histoire de Narcisse dans le Roman de la Rose.». Voir : Desmond et Sheingorn, op. cit., p. 277, n. 54.

${ }^{69}$ «Quant elle s'oï escondire / si en ot tel duel et tel ire / et le tint a si grand despit / qu'elle fu morte sanz respit. / Mes tot avant qu'ele morist / ele pria a Deu et requist / que Narcisus au cuer farasche, / qu'ele ot trové d'amer si lache / fust asproiez encore un jor / et destroiz d'autretele amor / dont il ne peüst mire atendre ; / [...] Ceste priere fu resnable / et por ce la fist Dex estable; » Guillaume de Lorris, Jean de Meun, Roman de la Rose, éd. F. Lecoy, Paris, Champion (Les Classiques du Moyen Âge, 92), 1970, 3 vol. , I, p. 45, v. 1451-1466.

${ }_{70}$ «Dames, cest essample aprenez / qui vers vos amis mesprenez; / car se vos les lessiez morir / Dex le vos savra bien merir. » Roman de la Rose, éd. cit., 1, p. 45, v. 1451-1466.

${ }^{71}$ Selon l'expression de J. Frappier dans l'article «Variations sur le thème du miroir, de B. de Ventadour à Maurice Scève », Histoire, mythes et symboles, Genève, Droz, 1976, p. 149-167.

${ }^{72}$ Epistre Othea, éd. cit., [16], p. 226, 1. 12.

${ }^{73}$ Ovide Moralisé, éd. cit., liv. III, p. 331, v. 1504-1508.

${ }^{74}$ Ovide Moralisé, éd. cit., liv. III, p. 339, v. 1909.

${ }^{75}$ Epistre Othea, éd. cit., [16], p. 226, 1. 17-18.

${ }^{76}$ On sait que le débat suscité par le Roman de la Rose a opposé Christine de Pizan à Jean de Montreuil, au cours des années 1401-1402. Sur cette polémique, voir: E. Hicks, Christine de Pizan, Jean Gerson, Jean de Montreuil, Gontier et Pierre Col. Le Débat sur le Roman de la Rose, Genève, Slatkine Reprints (Bibliothèque du XV siècle, 43), 1996.
} 
illustrations renforcent l'interprétation que le texte de l'Epistre propose de sa mort, ainsi que la condamnation portée contre l'orgueil. Dans le manuscrit Français 606, le motif iconographique évoquant le contexte offre au lecteur un indice des liens intertextuels qui déterminent à la fois la signification du texte [16] et celle de son illustration. Il joue un rôle essentiel dans la reconstruction du sens dont celle-ci est lestée. Il sollicite la culture du lecteur auquel il incombe de découvrir ce qui fait la cohérence de l'association entre l'image et le texte.

\section{Analyse de la figuration d'Athamas}

Dans les trois manuscrits étudiés, le texte [17] de l'Epistre et son illustration portent sur un sujet insolite : la crise de folie furieuse du roi Athamas (Ill. 3, 4, 6). Même si le livre IV de l'Ovide Moralisé renferme cette fable ${ }^{77}$, les enlumineurs ont rarement figuré explicitement la folie de ce roi dans les manuscrits illustrés. Certains ont représenté Ino, sur le point de se jeter dans la mer, portant un bébé emmailloté dans les bras ${ }^{78}$. Parfois, l'illustration montre Athamas tenant Léarchos par un pied, alors que Ino s'enfuit en emportant Mélicerte ${ }^{79}$. Même sous le couvert de la fable, les enlumineurs répugnent souvent à représenter ce qui pourrait porter atteinte au caractère sacré de l'autorité royale ${ }^{80}$.

Dans les manuscrits de l'Epistre, l'illustration, en accord avec le texte, présente Athamas comme le meurtrier de sa femme et de ses deux enfants. Cette interprétation de la fable se fonde sur une réécriture du texte de l'Ovide Moralisé, ou sur la reprise d'une version différente, mais non identifiée, de son histoire ${ }^{81}$. Le poète anonyme de l'Ovide Moralisé raconte comment Athamas, devenu fou sous l'effet des sortilèges de Tisiphone, a tué Léarchos en le jetant par terre et comment Ino, tenant Mélicerte dans les bras, a plongé dans la mer. Dans l'Epistre Othea, Christine, condense le récit du meurtre perpétré par Athamas :

Athamas occist la royne par rage et puis ses ij enfants ${ }^{82}$.

Alors que dans l'Ovide Moralisé, les maléfices de Tisiphone ont transformé les deux parents en meurtriers de leurs enfants, dans l'Epistre, le texte et l'image présentent Athamas comme l'unique auteur du triple meurtre.

Dans ce texte, Athamas est l'exemplum choisi par Christine pour mettre en garde le chevalier chrétien contre la colère, l'un des sept péchés capitaux. L'identification implicite de la colère à la folie est, au premier abord, surprenante car la fable des Métamorphoses reprise dans l'Ovide Moralisé présente le roi forcené

\footnotetext{
${ }^{77}$ Ovide Moralisé, éd. cit., liv. IV, p. 93-95, v. 3876-3963.

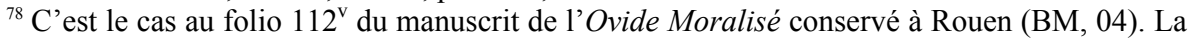
miniature est reproduite dans Desmond et Sheingorn, op. cit., p. 208, fig. 5. 8.

${ }^{79}$ Voir la reproduction du folio $53^{\mathrm{v}}$ du manuscrit Arsenal 5069 dans Desmond et Sheingorn, op. cit., p. 209, fig. 5.9.

${ }^{80} \mathrm{Il}$ n'en est plus ainsi à la fin du $\mathrm{XV}^{\mathrm{e}}$ et au $\mathrm{XVI}^{\mathrm{e}}$ siècles. Voir, par exemple, le folio $127^{\mathrm{v}} \mathrm{du}$ manuscrit de l'Ovide Moralisé, Copenhague, BR, Thott 399, copié vers 1480 ou l'édition de J. Steinman des Métamorphoses d'Ovide (Leipzig, 1582).

${ }^{81}$ Voir : Epistre Othea, éd. cit., p. 402, n. a.

${ }^{82}$ Epistre Othea, éd. cit., [17], p. 228, 1. 30-31.
} 
comme la victime impuissante de l'une des Furies, et non comme un homme ne sachant pas maîtriser ses passions.

Cependant, l'assimilation de la colère à la folie, fondée sur une analogie, était fréquente chez les moralistes antiques ${ }^{83}$. L'usage que l'un des devanciers de Christine $^{84}$ - Guillaume de Machaut - faisait de ce procédé d'écriture pour «dire apertement » au dédicataire du Confort d'Ami ce qu'il devait faire ${ }^{85}$, oriente notre attention vers une éventuelle signification politique du texte et de son illustration. La dénonciation de la colère s'inscrit d'ailleurs dans la tradition des Miroirs des princes. Dans le De Regimine Principum, Gilles de Rome fonde l'art de gouverner sur la sagesse et la vertu, le prince devant savoir se gouverner pour diriger le royaume avec sagesse. La vertu d' « attrempance »- la tempérance - lui permet de modérer ses passions, en éloignant les excès qui, rabaissant les hommes au rang des animaux, livrent le prince au mépris de ses sujets.

Le lien hypothétique du texte [17] et de son illustration avec le contexte politique se trouve confirmé par le texte [39] de l'Epistre qui rappelle que l'usage de " sors et de charmes et de enchantements pour avoir garison est chose deffendue et contre le commandement de sainte Eglise $^{86} »$. Cette allégation renvoie à la condamnation solennelle de la magie, prononcée le 19 septembre 1398 par le doyen de la faculté de théologie de Paris. Jean Gerson y dénonçait les erreurs des membres de la Cour qui, arguant qu'il est permis d'utiliser les arts magiques à de bonnes fins, avaient fait appel à des sorciers en vue de guérir le roi ${ }^{87}$. Des préoccupations politiques liées à la folie du roi Charles VI apparaissent ainsi, en filigrane, dans le texte de l'Epistre.

Si l'on insère la figuration d'Athamas dans ce contexte, le motif de l'enceinte fortifiée (Ill. 6) érige la représentation de la crise de folie meurtrière du roi Athamas en écho de la folie réelle frappant le roi de France. En présentant Athamas tel un acteur sur une scène de théâtre, l'illustration révèle publiquement la folie du roi. Christine outrepasse les tabous qui interdisaient aux chroniqueurs de la vie de Charles VI de nommer la maladie du roi ${ }^{88}$. Sous le voile de la fable, l'auteur prend la liberté d'adresser un avertissement au destinataire du manuscrit- le duc d'Orléans - en figurant symboliquement les menaces de destruction que la folie du roi fait peser sur le royaume. Espérant sauver la paix civile, Christine cherche sans doute à susciter une prise de conscience politique.

En invitant le duc d'Orléans à regarder en face la situation politique engendrée par la folie de Charles VI, Christine s'engage dans une démarche

\footnotetext{
${ }^{83}$ Horace, Epistre, I, 2, v. 62 : ira furor brevis ; Sénèque, De ira, I, I, 2 : Quidam itaque a sapientibus viris iram dixerunt brevem insaniam.

${ }^{84}$ Christine connaissait les œuvres de Guillaume de Machaut. Voir: Parussa, op. cit., [42], p. 417, n. b.

${ }_{85}$ Charles de Navarre, dédicataire du Confort d'Ami avait été fait prisonnier par le roi de France. Voir D. Lechat, Dire par fiction. Métamorphoses du «je» chez Guillaume de Machaut, Jean Froissart, Christine de Pizan, Paris, Champion (Etudes christiniennes, 7), 2005, p. 104.

${ }^{86}$ Epistre Othea, éd. cit., [39], p. 256, 1. 15-16.

${ }^{87}$ B. Guenée, La folie de Charles VI, Roi bien-aimé, [Paris], Perrin (Pour l'histoire), 2004, p. 79-98.

${ }^{88}$ Voir : Guenée, op. cit. , p. 28-34.
} 
audacieuse. Cependant, le recours à l'analogie laisse au destinataire toute liberté d'interpréter l'image, ce qui évite à l'auteur de déplaire au prince. Par ailleurs, le rôle joué dans la fable par Tisiphone tempère la hardiesse du dessein car, par la vertu de l'analogie, l'explication de la folie d'Athamas écarte les rumeurs imputant à Louis d'Orléans des tentatives d'empoisonnement de Charles VI ${ }^{89}$.

Ainsi, dans les manuscrits de Beauvais, Oxford et Paris, BNF, Fr. 606, les ressemblances entre les illustrations figurant Athamas étranglant ses deux enfants s'enracinent dans une version modifiée, choisie par Christine ou créée par ses soins, de l'histoire de ce roi. En vertu de l'assimilation de la colère à la folie, la représentation des conséquences tragiques de la folie furieuse du roi Athamas renforce le propos didactique stigmatisant la colère qui prive l'être humain de l'usage de sa raison : " De yre se doit moult garder ${ }^{90} »$ le chevalier chrétien.

Dans l'illustration du texte [17] (Ill. 6), le motif de l'enceinte fortifiée ajoute au sens premier du texte, des échos de l'actualité politique. Il fournit au lecteur un indice lui permettant d'identifier le contexte référentiel dans lequel l'image peut être décryptée. À l'instar de celle figurant Narcisse, cette illustration oriente le travail d'interprétation du lecteur vers la voie qui lui permettra de reconstruire la signification du rapport établi par l'auteur entre le texte et l'image. Dans les circonstances dans lesquelles Christine l'utilise, l'image remplit également une fonction « performative ${ }^{91}$ » en évoquant, sur le mode symbolique, la folie du roi. En brisant, par l'image, un tabou, Christine œuvre en vue d'une prise de conscience de la gravité de la situation politique du royaume. C'est la reconstruction, par le lecteur, du sens de l'association entre le texte et l'image qui lui permet de jouer ce rôle.

Par la mise en évidence des similitudes entre les trois manuscrits et celle des particularités iconographiques du manuscrit Français 606, l'étude comparée du cycle perdu et du cycle conservé apporte ainsi des clés d'interprétation des illustrations choisies par Christine pour ce manuscrit, destiné initialement, nous l'avons dit, à Louis d'Orléans.

Les deux manuscrits conservés à Beauvais et Oxford sont deux précieux témoins de l'existence d'un cycle iconographique de l'Epistre Othea, qui ornait sans doute un manuscrit aujourd'hui perdu. L'analyse comparée de ces quelques illustrations a souligné sa spécificité et l'éclairage que son étude apporte à l'interprétation du cycle conservé. Par ailleurs, l'étude du cycle perdu présente un intérêt supplémentaire. $\mathrm{Au} \mathrm{XVI} \mathrm{I}^{\mathrm{e}}$ siècle, des illustrations de l'édition Pigouchet - qui, nous l'avons dit, comporte des reflets de ce cycle - ont servi de modèles à l'imprimeur lyonnais Philippe le Noir et à Robert Wyer, imprimeur anglais ${ }^{92}$. Le

\footnotetext{
${ }^{89}$ Deux explications de la folie du roi étaient en présence : le recours au poison et l'usage de maléfices et de sortilèges. Voir: Guenée, op. cit., p. 79.

${ }^{90}$ Epistre Othea, éd. cit., [17], p. 228, 1. 41.

${ }_{91}$ Nous employons ce terme au sens que le philosophe anglo-saxon du langage, John Langshaw Austin lui accorde. Une énonciation « performative» ne décrit pas une réalité, mais elle exécute ("performs ») une action. Voir : J. L. Austin, Quand dire, c'est faire, tr. G. Lane, Éd. du Seuil (L'ordre philosophique), Paris, 1970, p. $42-56$ (éd. or. How to do things with words, 1962).

${ }^{92}$ Les planches gravées de la première édition Pigouchet, parue vers 1499, ont été reprises par cet imprimeur dans les éditions postérieures. Sur leur réutilisation par Philippe le Noir dans
} 
cycle iconographique perdu, partiellement distinct du cycle conservé dans les manuscrits princiers, a connu par l'intermédiaire de l'imprimerie, une large diffusion en France et en Angleterre. Ces images ont transmis à l'élite lettrée bourgeoise des préceptes moraux, énoncés à partir de l'allégorisation des mythes antiques. À ce titre, le cycle perdu a contribué de façon spécifique à la réception de l'œuvre.

Anne-Marie Barbier Université Charles-de-Gaulle Lille-3

son édition de 1522, voir: M. W. Driver, "Christine de Pizan and Robert Wyer: The .C. Hystoryes of troye, or l'Epistre Othea englished », Gutenberg Jahrbuch, 72, 1997, p. 128. Sur l'appropriation d'une partie des modèles de Pigouchet par R. Wyer, auteur et imprimeur d'une nouvelle traduction anglaise du texte, voir : Driver, art. cit., p. 125. 